\title{
High-order cumulants in the counting statistics of asymmetric quantum dots
}

Christian Fricke, Frank Hohls, Nandhavel Sethubalasubramanian, Lukas Fricke, and Rolf J. Haug

Citation: Appl. Phys. Lett. 96, 202103 (2010); doi: 10.1063/1.3430000

View online: https://doi.org/10.1063/1.3430000

View Table of Contents: http://aip.scitation.org/toc/apl/96/20

Published by the American Institute of Physics

\section{Articles you may be interested in}

Electron counting statistics and coherent states of electric current Journal of Mathematical Physics 37, 4845 (1996); 10.1063/1.531672

Electron counting of single-electron tunneling current

Applied Physics Letters 84, 2343 (2004); 10.1063/1.1691491

AC transport and full-counting statistics of molecular junctions in the weak electron-vibration coupling regime The Journal of Chemical Physics 146, 092313 (2017); 10.1063/1.4973707

Fast detection of single-charge tunneling to a graphene quantum dot in a multi-level regime Applied Physics Letters 101, 012104 (2012); 10.1063/1.4733613

Charge sensing in a Si/SiGe quantum dot with a radio frequency superconducting single-electron transistor Applied Physics Letters 101, 142103 (2012); 10.1063/1.4754827

Counting statistics in an InAs nanowire quantum dot with a vertically coupled charge detector Applied Physics Letters 100, 072110 (2012); 10.1063/1.3687198

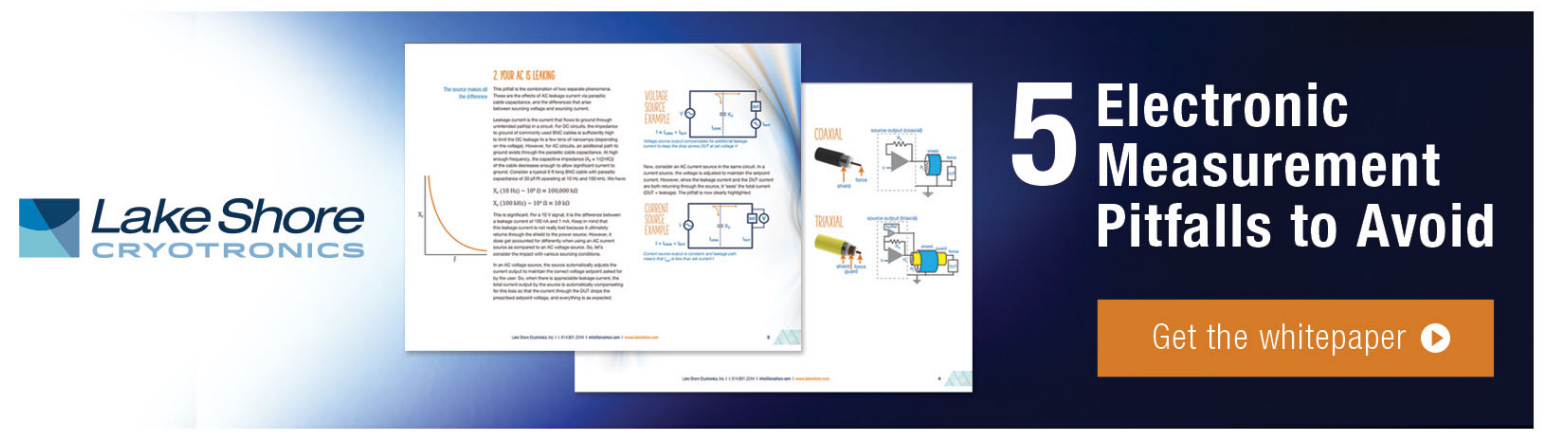




\title{
High-order cumulants in the counting statistics of asymmetric quantum dots
}

\author{
Christian Fricke, ${ }^{\text {a) }}$ Frank Hohls, Nandhavel Sethubalasubramanian, Lukas Fricke, and \\ Rolf J. Haug \\ Institut für Festkörperphysik, Leibniz Universität Hannover, Hannover, Niedersachsen 30167, Germany
}

(Received 25 November 2009; accepted 26 April 2010; published online 18 May 2010)

\begin{abstract}
Measurements of single electron tunneling through a quantum dot (QD) using a quantum point contact as charge detector have been performed for very long time traces with very large event counts. This large statistical basis is used for a detailed examination of the counting statistics for varying symmetry of the QD system. From the measured statistics we extract high order cumulants describing the distribution. Oscillations of the high order cumulants are observed when varying the symmetry. We compare this behavior to the observed oscillation in time dependence and show that the variation in both system variables lead to the same kind of oscillating response. () 2010 American Institute of Physics. [doi:10.1063/1.3430000]
\end{abstract}

Current fluctuations in mesoscopic systems allow to obtain information on transport that is not accessible from the average current alone. ${ }^{1}$ Measurements of the shot noise as a first step beyond the mean have been deployed successfully to examine correlations in the electron transport through a metallic island ${ }^{2}$ and through semiconducting quantum dot (QD) systems, ${ }^{3,4}$ but the extraction of higher moments directly from the current fluctuations is a demanding task ${ }^{5}$ and has not yet been achieved for transport through QDs. In contrast higher moments are naturally accessible in the context of full counting statistics (FCS). ${ }^{6,7}$ With the use of a quantum point contact (QPC) as a noninvasive charge detector with sufficiently fast time response, FCS became experimentally feasible in QD physics. ${ }^{8-11}$ In a measurement of the fourth and fifth cumulant complex behavior as a function of asymmetry and integration time with local minima was found. ${ }^{11} \mathrm{~A}$ significantly improved experimental technique has made the extraction of very high-order cumulants possible, revealing an oscillating behavior as function of integration time that strongly increases with the order of the moment. ${ }^{12,13}$ Such oscillations are also predicted theoretically in quantum optics ${ }^{15}$ and particle physics. ${ }^{14}$ The theoretical treatment has shown that these oscillations are a universally expected phenomenon for most physical systems and that in QDs they should show up prominently as function of the barrier asymmetry. ${ }^{12,16}$

In this paper, we present the experimental verification of the predicted oscillations in the higher cumulants as function of barrier asymmetry in a QD system. To this aim we employ real time single electron counting with a high bandwidth detector. This enables us to measure the FCS for electron transport through a QD on the microsecond-timescale, allowing us to gather an unprecedented large amount of counting events. With this large statistical basis we can examine the functional dependence of high order cumulants, which describe the statistical properties of the system, on the tunneling barrier asymmetry and also on the counting time. The observed oscillations as function of both parameters show large similarities that strongly support the universal nature of these oscillations.

${ }^{a)}$ Electronic mail: fricke@nano.uni-hannover.de.
Our device is based on a GaAs/AlGaAs heterostructure containing a two-dimensional electron system (2DES) $34 \mathrm{~nm}$ below the surface. The electron density is $\rho=4.6$ $\times 10^{15} \mathrm{~m}^{-2}$, and the mobility is $\mu=64 \mathrm{~m}^{2} / \mathrm{V} \mathrm{s}$. We have used an atomic force microscope (AFM) to define the QD and the QPC structure by local anodic oxidation of the surface ${ }^{17,18}$ the 2 DES below the oxidized surface is depleted and so insulating lines are defined.

An AFM image of our device is presented in Fig. 1. The dark lines depict the insulating oxide barriers written by the AFM. The QPC (left area) is separated from the QD structure (right area) by an insulating line. The QPC can be electrically tuned using the in-plane gate $G 3$. The current through the QPC is amplified by a current amplifier and detected in a time-resolved manner with a sampling rate of 500 $\mathrm{kHz}$. To achieve a high bandwidth charge detection, a custom low capacitance wiring is used to reduce technical noise caused by the input voltage noise of the amplifier. The bandwidth of the charge detection is only limited by the current
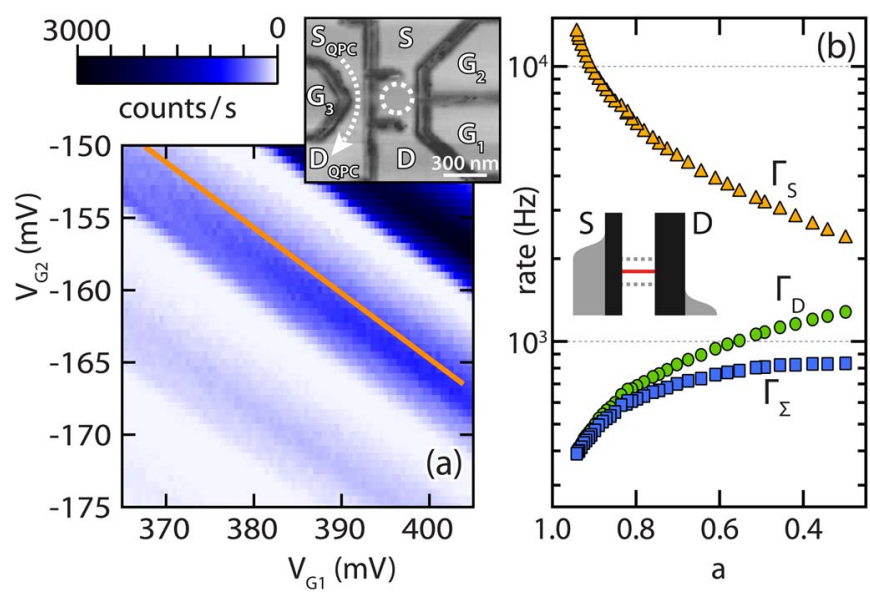

FIG. 1. (Color online) (a) Average current through the QD vs gate voltage at the in-plane gates G1 and G2. Current is measured counting electron by electron. A bias of $1.3 \mathrm{mV}$ is applied between source and drain contact of the QD. Inset: AFM image of the structure. (b) Measured tunneling rates $\Gamma_{S}$ (source), $\Gamma_{D}$ (drain), and the electron transport rate $\Gamma_{\Sigma}$ with respect to the asymmetry parameter $a$. The asymmetry parameter is modified by changing the QD gate configuration along the line shown in (a). 
amplifier bandwidth and slightly exceeds $100 \mathrm{kHz}$. The QPC bias is chosen sufficiently small to avoid back-action on the QD. ${ }^{19,20}$ The QD is coupled to source and drain electrodes via two tunneling barriers which can be separately controlled with gate voltages $V_{G 1}$ and $V_{G 2}$. These gates are also used to tune the number of electrons on the QD. The sample is placed in a $\mathrm{He}^{3}$ refrigerator reaching temperatures down to $400 \mathrm{mK}$.

Setting the device to a situation where electrons can enter and leave the QD results in a fluctuating charge on the QD. The electron number on the dot is sequentially changing from $\mathrm{N}$ to $\mathrm{N}+1$ and from $\mathrm{N}+1$ to $\mathrm{N}$ electrons. This leads to a corresponding change in the potential at the QPC which has a working point on the edge of the first conductance step. Thus the QPC acts as a sensitive charge detector as each change in the potential due to a changing electron number on the dot leads to a current change at the QPC.

The average current versus $V_{G 1}$ and $V_{G 2}$ as measured by single electron counting is shown in Fig. 1(a). A bias of 1.3 $\mathrm{mV}$ is applied to the QD. The white areas without electron tunneling events correspond to Coulomb blockade with fixed electron number on the QD. In between wide stripes with a significant number of tunneling events are observed due to the applied QD bias voltage. The charging energy of the QD is $1.6 \mathrm{meV}$, the single particle level spacing is $0.27 \mathrm{meV}$. The nonzero electron temperature leads to an ambiguous tunneling direction at the edges of these stripes where the Fermilevel of a lead is in resonance with the dot level.

To analyze only unidirectional transport, the system is tuned to a situation where the transport state of the dot is sufficiently far from the source and drain Fermi energies, thus allowing no back-tunneling from the dot to the source contact or from drain to the dot [compare inset in Fig. 1(b)]. The symmetry of the tunneling rates are studied using both QD gates to keep the transport in the unidirectional regime and concurrently to change the asymmetry of the tunneling barriers. The line drawn in Fig. 1(a) indicates the gate voltages used in the following. The resulting tunneling rates are displayed in Fig. 1(b) with respect to the asymmetry factor $a=\left(\Gamma_{S}-\Gamma_{D}\right) /\left(\Gamma_{S}+\Gamma_{D}\right)$ with $\Gamma_{S}$ and $\Gamma_{D}$ the tunneling rates between source drain and the QD, respectively. For smaller $V_{G 1}$ and more positive $V_{G 2}$ [upper left corner of Fig. 1(a)] the system is rather asymmetric with $\Gamma_{S} \approx 20 \mathrm{kHz}$ and $\Gamma_{D}$ $\approx 0.2 \mathrm{kHz}$. Following the line $\Gamma_{S}$ drops down to $2.3 \mathrm{kHz}$ and $\Gamma_{D}$ increases to $1.2 \mathrm{kHz}$. Thus the asymmetry factor $a$ is varied from 0.98 to 0.3 . The total rate of electron transfer $\Gamma_{\Sigma}=\Gamma_{S} \times \Gamma_{D} /\left(\Gamma_{S}+\Gamma_{D}\right)$ reaches its highest value $\Gamma_{\Sigma}$ $=0.9 \mathrm{kHz}$ at $a=0.3$ and drops with increasing asymmetry $a$.

The detection of individual tunneling events allows us to extract the FCS of electron transport through the QD. Before going into this analysis it is useful to introduce a dimensionless time $\Gamma_{S} \times t$ which is equivalent to setting $\Gamma_{S}$ equal to unity. This is necessary to separate time and asymmetry dependence as according to Ref. 12 time dependent oscillations are universal on the timescale $\Gamma_{S} \times t$. Then the system is solely governed by the asymmetry parameter $a$. Thus the statistical properties are also completely determined by this single system parameter $a$. Of course we have to note that in our experiment another rate exists, namely, the detector bandwidth $\Gamma_{\operatorname{det}}$. However, if we fix this rate relatively to $\Gamma_{S}$ we again have a system whose statistical properties are solely varied by the asymmetry. We realize this fixation of
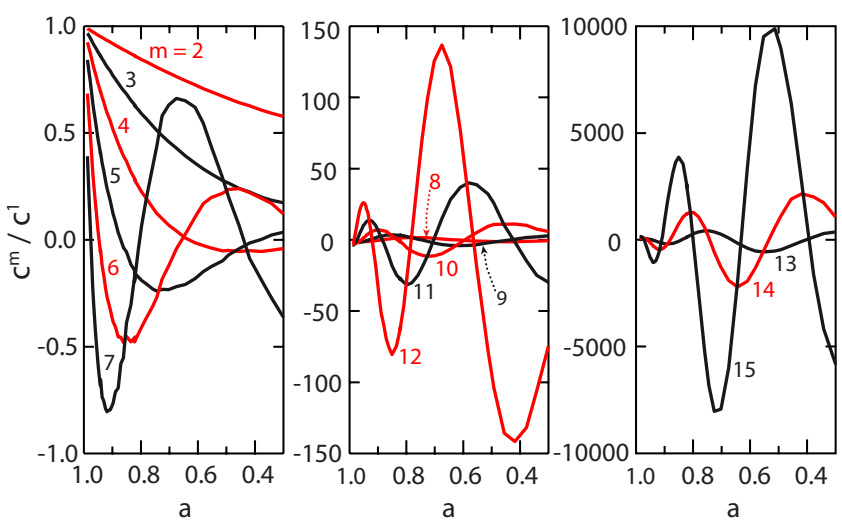

FIG. 2. (Color online) Cumulants of order $m=2, \ldots, 15$ as a function of asymmetry factor $a$. While the low order cumulants show a monotonic behavior the cumulants with order $m>4$ reveal distinct oscillations with factorial increase in amplitude (up to 10000 for the 15th cumulant). The cumulants are determined for a constant dimensionless counting time interval $\Gamma_{S} \times t_{0}=3.2$.

the detector bandwidth by a postselection of detected events with an artificial detector bandwidth of $\Gamma_{\mathrm{det}}=4 \Gamma_{S}$.

We will now examine the counting statistics of the QD. This can be done either by direct analysis of the whole probability distribution of the number of transferred electrons $n$ in a certain time interval or more common by the cumulants $c_{m}$ of this distribution. The first cumulant is the mean of the number of transferred electrons, $c_{1}=\langle n\rangle$, the second, $c_{2}$, is the variance, and the third is the skewness. Cumulants of higher order $m$ are very sensitive to the details of the transport process. Recently it was found experimentally that the high-order cumulants of QD transport oscillate as function of the counting time interval. ${ }^{12}$ In the same paper, it is stated that such oscillations are expected universally for nearly any system when changing a relevant parameter, e.g., the symmetry of the tunneling rates in a QD system. ${ }^{12}$ For our experimental study of this predicted symmetry dependence of high-order cumulants we varied the asymmetry parameter $a$ and measured for each $a$ about one million tunneling events. This statistical sample enables us to determine cumulants up to the 15 th order with high precision.

In Fig. 2 the normalized cumulants $c^{m} / c^{1}$ are shown as function of the asymmetry factor $a$ for $m=2, \ldots, 15$. For high asymmetry $(a \rightarrow 1)$ all normalized cumulants start from a value of 1 due to the Poissonian limit of a single dominant barrier. The first cumulants then drop monotonically toward the symmetric limit. For $m \geq 4$ a more complicated behavior can be observed. Beginning with the fourth order cumulant a minimum starts to develop, shifting to higher asymmetry and increasing in amplitude with rising order. For the sixth order an additional maximum appears that also gets more prominent with rising order of the cumulants. This trend continues for even higher cumulants, the number of oscillation increases and also the amplitude rises dramatically. For the 12th order values up to 145 are reached and the amplitude of the 15 th cumulant already exceeds 10000 . This factorial increase was shown already for oscillations as function of time $^{12}$ and appears here again, showing the universal nature of the oscillations.

The asymmetry dependence shown in Fig. 2 was evaluated for a certain choice of the dimensionless length of the counting interval. We can also determine the dependence on 


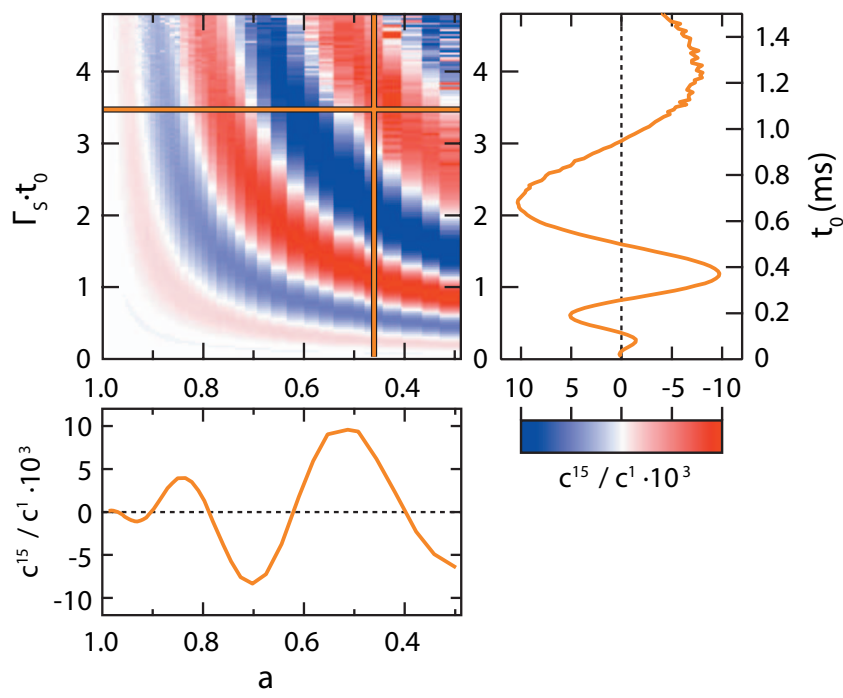

FIG. 3. (Color) Time and symmetry dependence for the 15th cumulant. Oscillations can be observed for varying time base $\Gamma_{S} \times t_{0}$ and symmetry $a$. Two profile lines are shown displaying the substantial similarity of both types of oscillations.

the dimensionless counting interval for each value of the asymmetry $a$, thereby mapping out the cumulants as function of both parameters. The result obtained for the 15 th cumulant $c_{15}$ is shown in Fig. 3(a). The figure nicely reveals the similarity in the dependencies on asymmetry and on dimensionless time-the general features are nearly symmetric with respect to the diagonal. For a further illustration of these similarities we plot two profiles in Figs. 3(b) and 3(c), taken at fixed asymmetry respect to the fixed normalized time. Both curves, $c_{15}$ as a function of time $\Gamma_{S} \times t_{0}$ and for $c_{15}$ as a function of asymmetry $a$, show oscillations of very similar shape and amplitude. This correspondence between the different parameters nicely demonstrates that the occurrence and the strength of these oscillations are of universal nature. ${ }^{12}$ It is also interesting to examine the behavior at long times or toward the case of symmetric rates, $a=0$. For long times the dependence on $\Gamma_{S} \times t_{0}$ becomes weak and in the long time limit the cumulants will become sole function of the asymmetry. Toward $a=0$ the dependence on asymmetry flattens out and the cumulants depend mainly on time. These transitions again reveal the similarities in the dependence on these different parameters and support the underlying universal nature of the oscillatory behavior.

In conclusion, we have realized a high-bandwidth detection of single electron transport in a QD with a sufficiently large event number to examine the symmetry dependence of the cumulants of the counting probability distribution up to the 15 th order. We have verified the theoretically predicted oscillation of the high order cumulants as function of the QD barrier asymmetry. Furthermore we have performed a twodimensional-parameter study of the cumulants as function of both asymmetry and time. Our experimental results nicely reveal the similarity of the dependence on these different parameters, thereby demonstrating the underlying universal nature of the cumulant oscillations.

We thank C. Flindt for many fruitful discussions. The work was supported by the Federal Ministry of Education and Research of Germany via nanoQUIT and the German Excellence Initiative via QUEST.

${ }^{1}$ Y. M. Blanter and M. Buttiker, Phys. Rep. 336, 1 (2000).

${ }^{2}$ H. Birk, M. J. M. de Jong, and C. Schöneberger, Phys. Rev. Lett. 75, 1610 (1995).

${ }^{3}$ A. Nauen, I. Hapke-Wurst, F. Hohls, U. Zeitler, R. J. Haug, and K. Pierz, Phys. Rev. B 66, 161303 (2002).

${ }^{4}$ A. Nauen, F. Hohls, N. Maire, K. Pierz, and R. J. Haug, Phys. Rev. B 70, 033305 (2004).

${ }^{5}$ B. Reulet, J. Senzier, and D. E. Prober, Phys. Rev. Lett. 91, 196601 (2003)

${ }^{6}$ D. A. Bagrets and Yu. V. Nazarov, Phys. Rev. B 67, 085316 (2003).

${ }^{7}$ L. S. Levitov, H. Lee, and G. B. Lesovik, J. Math. Phys. 37, 4845 (1996).

${ }^{8}$ S. Gustavsson, R. Leturcq, B. Simovic, R. Schleser, T. Ihn, P. Studerus, K. Ensslin, D. C. Driscoll, and A. C. Gossard, Phys. Rev. Lett. 96, 076605 (2006).

${ }^{9}$ T. Fujisawa, T. Hayashi, R. Tomita, and Y. Hirayama, Science 312, 1634 (2006)

${ }^{10}$ C. Fricke, F. Hohls, W. Wegscheider, and R. J. Haug, Phys. Rev. B 76, 155307 (2007).

${ }^{11}$ S. Gustavsson, R. Leturcq, T. Ihn, K. Ensslin, M. Reinwald, and W. Wegscheider, Phys. Rev. B 75, 075314 (2007).

${ }^{12}$ C. Flindt, C. Fricke, F. Hohls, T. Novotný, K. Netocný, T. Brandes, and R. J. Haug, Proc. Natl. Acad. Sci. U.S.A. 106, 10116 (2009).

${ }^{13}$ C. Fricke, F. Hohls, C. Flindt, and R. J. Haug, Physica E (Amsterdam) 42, 848 (2010).

${ }^{14}$ I. M. Dremin and R. C. Hwa, Phys. Rev. D 49, 5805 (1994).

${ }^{15}$ V. V. Dodonov, I. M. Dremin, P. G. Polynkin, and V. I. Man'ko, Phys. Lett. A 193, 209 (1994).

${ }^{16}$ C. Flindt, T. Novotný, A. Braggio, M. Sassetti, and A. Jauho, Phys. Rev. Lett. 100, 150601 (2008)

${ }^{17}$ R. Held, T. Vancura, T. Heinzel, K. Ensslin, M. Holland, and W. Wegscheider, Appl. Phys. Lett. 73, 262 (1998).

${ }^{18}$ U. F. Keyser, H. W. Schumacher, U. Zeitler, R. J. Haug, and K. Eberl, Appl. Phys. Lett. 76, 457 (2000).

${ }^{19}$ V. S. Khrapai, S. Ludwig, J. P. Kotthaus, H. P. Tranitz, and W. Wegscheider, Phys. Rev. Lett. 97, 176803 (2006).

${ }^{20}$ S. Gustavsson, M. Studer, R. Leturcq, T. Ihn, K. Ensslin, D. C. Driscoll, and A. C. Gossard, Phys. Rev. Lett. 99, 206804 (2007). 